\title{
ELECTROLYSIS-BASED PARYLENE-BALLOON ACTUATORS
}

\author{
L. Giacchino* and Y.C. Tai \\ California Institute of Technology, Pasadena, California, USA
}

\begin{abstract}
This work reports the design, fabrication, testing and modeling of electrolysis-based fully encapsulated parylene balloon actuators. The proposed actuators have the advantage of high force at low power and low voltage. The main issues are low actuation speed and limited number of actuation cycles due to loss of water by permeation through the balloon membrane. In our case, the actuators are targeted to move neuroprobes after implantation, where slow movement is acceptable but low power consumption is of demand.
\end{abstract}

\section{KEYWORDS}

Electrolysis, actuators, parylene, permeation

\section{INTRODUCTION}

Electrolysis as a method of actuation has been already demonstrated [1,2], and it has been employed in various applications, such as electrolysis pumps for microfluidics [3] and actuation of movable neural probes [4]. The main advantage of this kind of actuators is their ability to provide large force and displacement, while requiring low actuation voltage and low power. The principle is based on the conversion of liquid water into gas (i.e., hydrogen and oxygen), which provides a dramatic increase in volume (if the gas is free to expand) or in pressure (if the volume is fixed), or a combination thereof.

Water electrolysis proceeds with the following wellknown half-reactions at the electrodes. If protons carry the charge, the reactions are

$$
\begin{aligned}
& \text { anode: } 2 \mathrm{H}_{2} \mathrm{O}(\mathrm{l}) \rightarrow \mathrm{O}_{2}(g)+4 \mathrm{H}^{+}(a q)+4 e^{-} \\
& \text {cathode: } 4 \mathrm{H}^{+}(a q)+4 e^{-} \rightarrow 2 \mathrm{H}_{2}(g) \\
& \text { total: } 2 \mathrm{H}_{2} \mathrm{O}(l) \rightarrow 2 \mathrm{H}_{2}(g)+\mathrm{O}_{2}(g)
\end{aligned}
$$

The actuation can be reversed in two ways: by letting hydrogen and oxygen recombine into water (by means of a suitable catalyst, like platinum), or by letting the gases escape from the volume of the actuator.

\section{OVERVIEW}

The actuators presented in this paper use a parylene balloon to enclose an electrolyte solution. Two platinum electrodes are used to perform water electrolysis inside the balloon.

The devices consist of a silicon cross over which a balloon is fabricated. The cross serves as a substrate for the platinum electrodes, and it provides two lateral beams which help contain the sacrificial photoresist used during balloon fabrication (see Fig. 1.a).

In operation, the generated gases cause an increase in pressure inside the balloon, which in turns causes the balloon to expand. When electrolysis is stopped, the balloon relaxes because of two concurrent phenomena: the recombination of gases back into water, and the permeation of gases through the parylene balloon membrane. As a matter of fact, parylene is known for its good diffusion barrier properties compared to other polymers, but it still has significant permeability to gases and water especially when the surface-to-volume ratio is high for the balloon.

We have built electrolysis balloon devices and tested them experimentally. We have also performed theoretical analysis on the experimental results. The results show that permeation dominates the relaxation behavior in our devices, which bears important consequences on the duration of an actuation cycle and on the lifetime of the device.

\section{FABRICATION \\ Silicon cross}

The fabrication of the silicon cross is illustrated in Fig. 1 , steps $1-5$. To begin with, $200 \mu \mathrm{m}$ thick wafers are used for the convenience of etching and release of the devices.

A first layer of parylene $\mathrm{C}, 0.5 \mu \mathrm{m}$ thick, is deposited by room-temperature LPCVD. The parylene is then molten at $350{ }^{\circ} \mathrm{C}$ in a nitrogen environment, in order to prevent oxidative degradation of the polymer.

A second layer of parylene $\mathrm{C}, 1 \mu \mathrm{m}$ thick, is deposited on top of the molten parylene layer. The molten parylene acts as an adhesion layer between silicon and this second parylene layer. Platinum is then deposited by e-beam evaporation, and it is patterned by a liftoff process. No adhesion layer is used between platinum and parylene, because metals commonly used as adhesion layers (like titanium) are easily corroded during electrolysis. The adhesion of platinum to parylene is sufficient for the fabrication of the devices.

Parylene is patterned by reactive ion etching (RIE) with oxygen plasma, using a photoresist mask. The same mask is used for the following deep reactive ion etching (DRIE) step, which defines the shape of the devices and releases them from the wafer. Fig. 2.a shows one of the fabricated structures.

\section{Parylene balloon}

The fabrication of the parylene balloon is illustrated in Fig. 1, steps 6-10. The silicon structure is mounted on a 

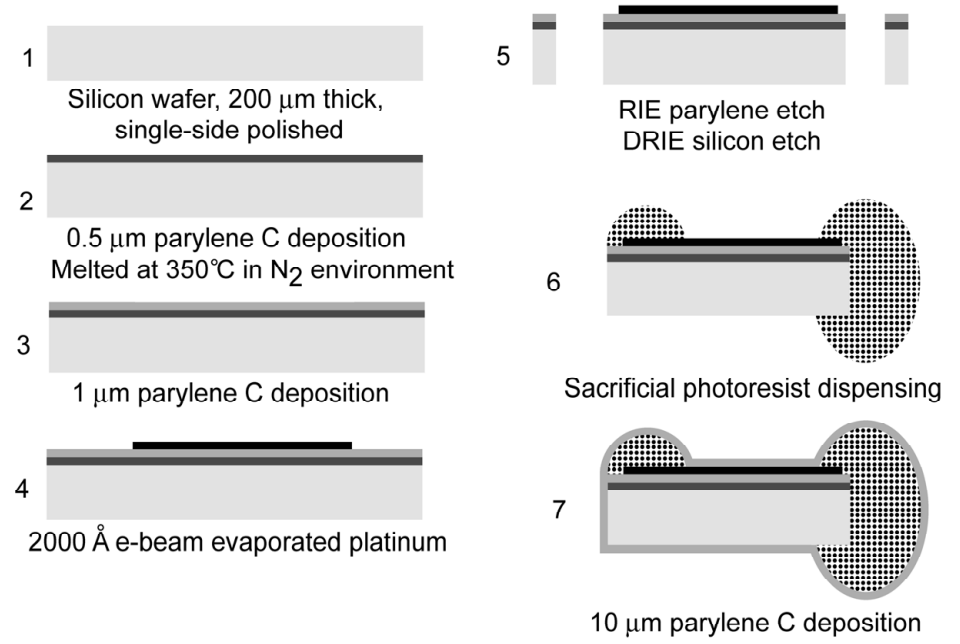

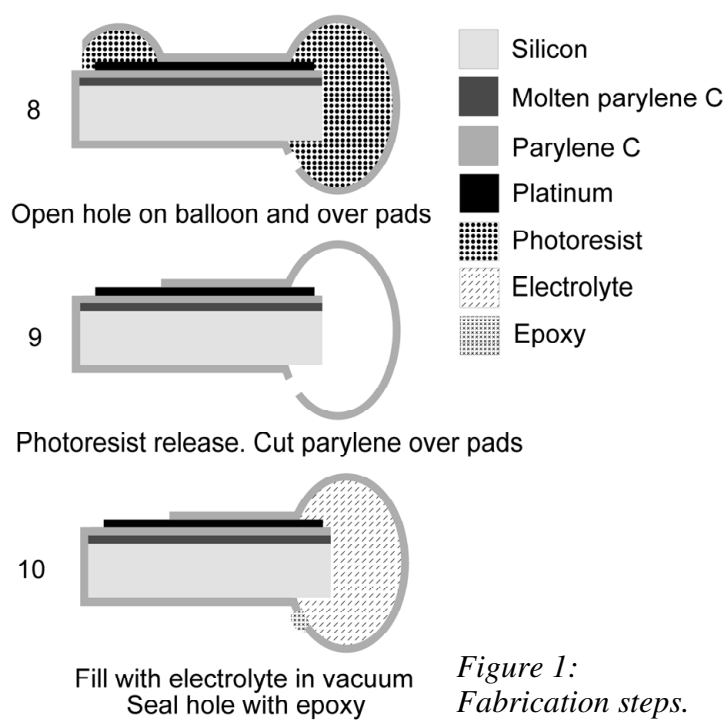

Fabrication steps. glass slide, for ease of handling. Sacrificial photoresist (AZ4620) is dispensed over the silicon structure to define the balloon shape. A commercial fluid dispenser (EFD 2000XL) with a 33-gauge needle was used. Some photoresist is dispensed over the pads as well, so they can be reopened after parylene deposition.

The photoresist was baked on a hotplate at $70{ }^{\circ} \mathrm{C}$. It is important to make sure that no gas bubbles are trapped in the photoresist, as they may burst in vacuum during the following parylene deposition. The baking temperature cannot be raised over $70{ }^{\circ} \mathrm{C}$, in order to limit photoresist reflow, which tends to make the balloon assume a spherical shape. The most desirable shape for the balloon is an ellipsoid with the shortest axis along the displacement direction, so that the internal pressure is better exploited to produce displacement and force in that direction.

A layer of parylene is then deposited. Due to the conformal nature of the deposition, the sacrificial photoresist is sealed. A sharp needle is used to poke a hole in the parylene (the needle can be heated to simplify the process) on the balloon and over the pads. The device is then immersed in acetone, for photoresist release.

After photoresist is completely released (see Fig. 2.b), and no acetone is left in the interior of the balloon, the device is immersed in an electrolyte solution inside a vacuum chamber. The vacuum causes the air inside the balloon to expand out of the balloon through the hole. When vacuum is broken, electrolyte is driven into the balloon, filling it. The balloon is finally sealed with a small drop of epoxy.

\section{Silicon dises}

Two circular silicon discs are mounted on some devices to define the balloon shape more accurately (Fig. 2.c). One disc has a central hole which mates with the silicon beam supporting the electrodes. Some sacrificial photoresist is then dispensed over this disc. A second disc (without a hole) is positioned on top of the photoresist. The space between the two discs is then completely filled with photoresist. The discs are fabricated by DRIE, using thermally-grown silicon dioxide as a mask.

\section{TESTING SETUP}

To measure the displacement and force provided by the balloons, a linear variable differential transformer (LVDT) is used. The LVDT is spring loaded, and its spring constant has been measured to be $2.5 \mathrm{mN} / \mu \mathrm{m}$. Therefore, the measured displacement is lower than it would be in free conditions, as the actuator has to overcome the spring force.

Electrolysis is driven by a voltage source, and two multimeters are used to measure the applied voltage and the resulting current. The data from the LVDT and multimeters is acquired by LabVIEW on a computer, and exported to Matlab for analysis.

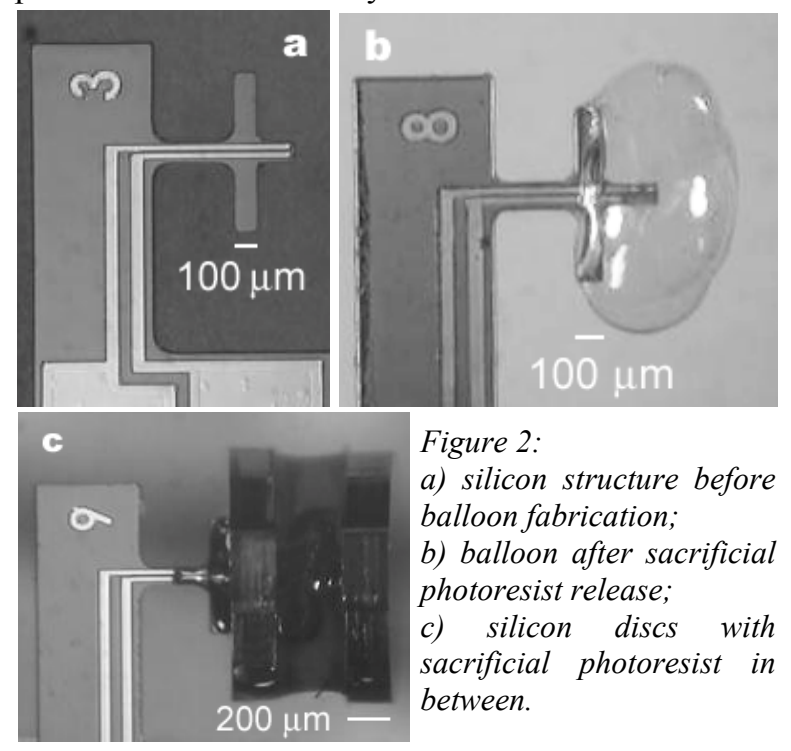



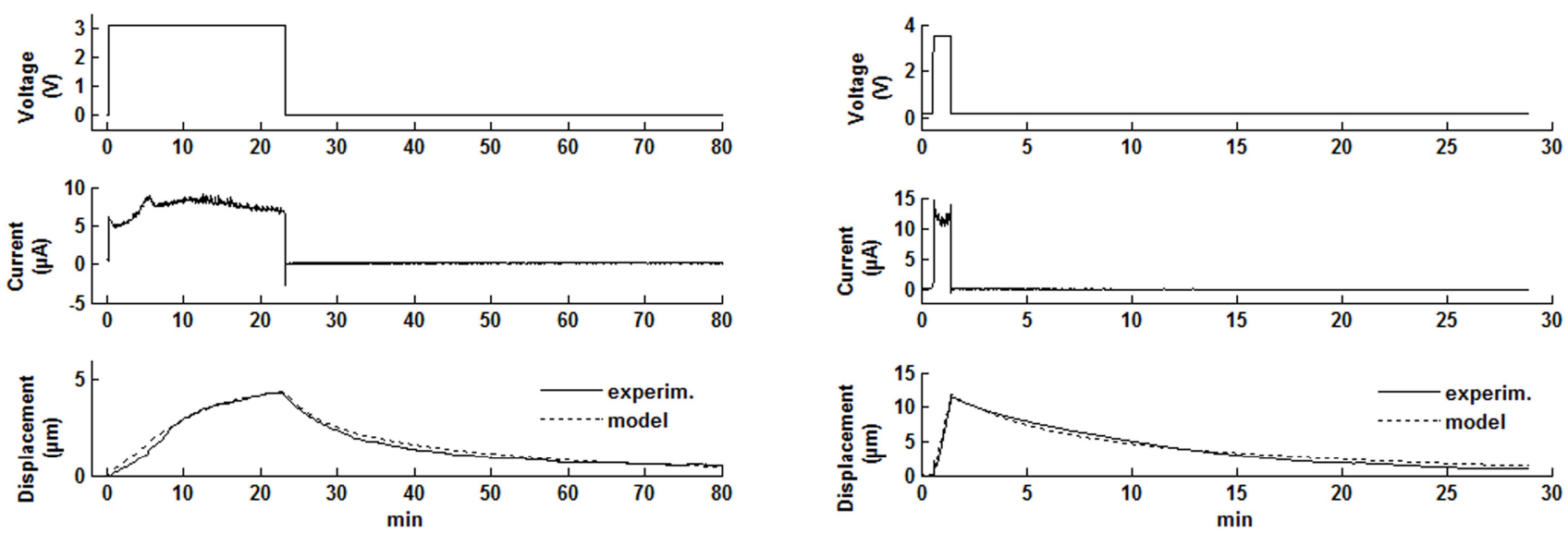

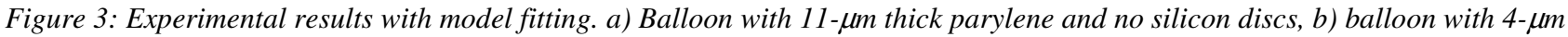
thick parylene and silicon discs.

\section{RESULTS}

Experimental results for the actuation and relaxation of balloon actuators are shown in Fig. 3. In the case of Fig. 3.a the balloon has a parylene membrane thickness of $11 \mu \mathrm{m}$, and the electrolyte used is a 95:5:0.1 solution of deionized water, acetic acid and methanol. No silicon discs are used in this device. The maximum displacement obtained is about $4.5 \mu \mathrm{m}$, corresponding to a force of $11.25 \mathrm{mN}$.

In the case of Fig. 3.b the balloon has a parylene membrane thickness of $4 \mu \mathrm{m}$, with an additional layer of PDMS about $50 \mu \mathrm{m}$ thick for mechanical reinforcement. PDMS is much more permeable than parylene, so it does not significantly affect the total permeability. The electrolyte used in this case is a $0.25 \mathrm{M}$ solution of $\mathrm{MgSO}_{4}$. The maximum displacement obtained is $11.5 \mu \mathrm{m}$, corresponding to a force of $28.75 \mathrm{mN}$. The displacement rate is higher than in the first case due to the presence of the silicon discs, and the relaxation time is shorted due to the thinner parylene membrane.

\section{THEORETICAL ANALYSIS Mathematical model}

A model is developed to estimate the internal pressure in the balloon as a function of time. The internal pressure in turns enables us to calculate the balloon displacement and force.

Three mechanisms taking place inside the actuator are included in the model: generation of oxygen and hydrogen from water, recombination of oxygen and hydrogen back into water, and permeation of oxygen, hydrogen and water through the balloon wall. Since the experiments are run in air, permeation of nitrogen through the balloon wall is included as well.

The model keeps track of the amount of water, hydrogen, oxygen and nitrogen inside the balloon as a function of time by means of mass balance equations

$$
\begin{aligned}
& d n_{\mathrm{O} 2}(t) / d t=g(t)-r(t)-f_{\mathrm{O} 2}(t) \\
& d n_{\mathrm{H} 2}(t) / d t=2 g(t)-2 r(t)-f_{\mathrm{H} 2}(t) \\
& d n_{\mathrm{H} 2 \mathrm{O}}(t) / d t=-2 g(t)+2 r(t)-f_{\mathrm{H} 2 \mathrm{O}}(t) \\
& d n_{\mathrm{N} 2}(t) / d t=-f_{\mathrm{N} 2}(t)
\end{aligned}
$$

where $n_{x}(t)$ is the number of moles of component $x$ inside the balloon at time $\mathrm{t}$ :

Each term on the right side of the equations models one of the mechanisms mentioned above: $g(t)$ and $r(t)$ respectively represent generation and recombination of oxygen and hydrogen, while $f_{x}(t)$ represents the permeation of component $x$ through the balloon membrane. The terms $g(t)$ and $r(t)$ need a multiplicative factor of 2 when it accounts for the stoichiometry of the reaction, like for the hydrogen in $2 \mathrm{H}_{2}+\mathrm{O}_{2} \leftrightarrow 2 \mathrm{H}_{2} \mathrm{O}$.

The generation term $\mathrm{g}(\mathrm{t})$ is considered proportional to the electrolysis current through a charge efficiency term $\varepsilon$. The recombination term $\mathrm{r}(\mathrm{t})$ is modeled by a simple thirdorder reaction kinetics equation.

Permeation through the balloon membrane for each component is directly proportional to the balloon membrane surface area $\mathrm{A}$ and inversely proportional to its thickness h. For the components in the gas phase, permeation is assumed to be proportional to the difference in partial pressure $\mathrm{p}$ between the interior and exterior of the balloon membrane

$$
f_{x}(t)=c_{x} \frac{A}{h} \Delta p_{x}
$$

The proportionality constant $c_{x}$ is the permeability coefficient of component $\mathrm{x}$ through parylene. Permeation of liquid water is assumed proportional to the pressure of its saturated vapor (for which data is commonly available in literature), as they have the same chemical potential. Since the saturated vapor pressure of water does not vary significantly with the total pressure in the system, water permeation is considered independent of pressure. 
The balloon contains two separate phases, a liquid one and a gaseous one, which share the total volume of the balloon. The two phases are considered to be always at equilibrium with each other, so the chemical potential of each component is the same in both phases. This is the reason why permeation for each component is assumed to occur over the whole surface area of the balloon. However, in terms of composition, the liquid phase can be assumed to contain only water, and the gas phase can be assumed to contain only a mixture of hydrogen, oxygen and nitrogen, because the amount of intermixing between the two can be neglected with good approximation.

The volume of the liquid phase is easily computed (water is assumed to be incompressible). The volume of the gas phase $\mathrm{V}_{\text {gas }}$ is thus the difference of the balloon volume and the volume of liquid phase. If the components of the gas phase are behave like a mixture of ideal gases, the total pressure in the balloon is the sum of the partial pressure of each component in the phase and we can write

$$
\begin{aligned}
P(t) & =p_{\mathrm{H}_{2} \mathrm{O}}(t)+p_{\mathrm{O}_{2}}(t)+p_{N_{2}}(t)= \\
& =\frac{n_{\mathrm{H}_{2} \mathrm{O}}(t)+n_{\mathrm{O}_{2}}(t)+n_{N_{2}}(t)}{V_{\text {gas }}(t)} R T
\end{aligned}
$$

All the model equations are solved simultaneously using a finite-difference method implemented in Matlab.

\section{Application of the model}

The model has been applied to fit experimental results. Values provided by our parylene supplier (Specialty Coating Systems) were used as a starting point for the fitting of the permeability coefficients. The current supplied to the electrodes as a function of time was taken from the experimental data and supplied to the model.

The results are shown in Fig. 3 together with the experimental data. For both experiments the fitting gives an electrolysis charge efficiency $\varepsilon=10 \%$. A possible explanation for the low efficiency is recombination of oxygen and hydrogen at the electrodes immediately after generation by electrolysis, due to the small separation (10 $\mu \mathrm{m})$ between the electrodes and to the catalytic action of platinum.

The model predicts that the pressure inside the balloon at the point of maximum displacement is $2.5 \mathrm{~atm}$ for Fig. 3.a and $0.22 \mathrm{~atm}$ for Fig. 3.b (measured over external atmospheric pressure). This confirms the role of the silicon discs in enhancing displacement and force in the actuation direction.

The model shows that the relaxation of the balloon is dominated by permeation of oxygen and hydrogen through the balloon membrane. The recombination rate is slow due to the small platinum electrode area relative to the total volume of the balloon. It is then permeation that determines the balloon relaxation speed.

Also, due to permeation there is a net loss of water over time, which limits the lifetime of the actuators. The model estimates a liquid water volume loss equal to about $0.5 \%$ of the total balloon volume during both experiments shown in Fig. 3.

\section{APPLICATIONS}

Applications requiring relatively large actuation force or displacement at very low power, and where actuation speed is not of demand, are ideal for the balloon actuators. One such application is the actuation of movable neuroprobes. Two balloon actuators can be combined into a bidirectional inchworm mechanism, in which one balloon is used to contact the probe and the other one to move it. These devices are currently under test, and they will be presented in a future paper.

\section{CONCLUSIONS}

Parylene-balloon electrolytic actuators have been designed, fabricated and tested. The actuators require very low power to operate, in the order of tens of $\mu \mathrm{W}$, and they can provide force in the order of tens of $\mathrm{mN}$. A model has been developed which closely fits the experimental data.

Some aspects of the actuators need further optimization, in particular actuation speed, lifetime and electrolysis efficiency. Actuation speed can be enhanced by faster recombination or permeation, while lifetime can be extended by exploiting osmosis to drive water back into the balloon when the actuators are employed in an aqueous environment. A more careful design of the electrodes may also improve the electrolysis efficiency. All these optimizations are currently under way in our laboratory.

\section{ACKNOWLEDGEMENTS}

The funding of the work is provided by NIH, under Award Number R01 EY015545.

\section{REFERENCES}

[1] C.G. Cameron, M.S. Freund, "Electrolytic actuators: Alternative, high-performance, material-based devices", in PNAS 2002, pp. 7827-7831.

[2] C. Pang, Y.C. Tai, J.W. Burdick, R.A. Andersen, "Electrolysis-based diaphragm actuators", Nanotechnology, vol. 17, pp.64-68.

[3] J. Xie, Q. Hie. Y.C. Tai, J. Liu, T. Lee, "Electrolysisbased on-chip dispensing system for ESI-MS", in IEEE MEMS 2003, Jan 19-23, 2003, pp. 443-446.

[4] C. Pang, Y.C. Tai, J.W. Burdick, R.A. Andersen, "Electrolysis-based Parylene Balloon Actuators for Movable Neural Probes", in IEEE NEMS 2007, Jan 16-19, 2007, pp. 913-916.

\section{CONTACT}

* L. Giacchino, tel: +1-626-395-5899;

lucagiac@caltech.edu 